\title{
Low Cost 100 Gbps Multicore VCSEL Based Transmitter Module Platform
}

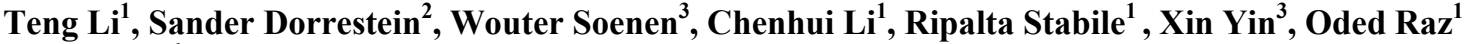 \\ ${ }^{1}$ Institute for Photonic Integration, Eindhoven University of Technology, Eindhoven, the Netherlands \\ ${ }^{2}$ TE Connectivity, Hertogenbosch, the Netherlands \\ ${ }^{3}$ Ghent University-iMinds-imec, Belgium \\ Email:T.Li@tue.nl
}

\begin{abstract}
We demonstrate a flexible printed circuit board platform for a $100 \mathrm{Gbps}$ VCSEL based transmitter with direct light launching into a multi-core-fiber using a 3D glass interposer as fanout Error free operation is demonstrated.

OCIS codes: (060.0060) Fiber optics and optical communications; (060.2360) Fiber optics links and subsystems
\end{abstract}

\section{Introduction}

The required interconnect bandwidth for data center network is increasing exponentially [1]. Many of the tier one data center operators are looking to move to single mode based optical interconnects [2]. Increasing capacity for single mode based transmission systems has long been achieved using DWDM [3]. Most DWDM solutions rely on edge emitters and planar integrated circuits which require very tightly controlled coupling schemes [4]. Also in most cases DFB based solutions require relatively high biasing current to allow direct current modulation at speeds above $10 \mathrm{Gbps}$ and are therefore often co-integrated with external modulators. This makes these solutions expensive, power hungry and technically challenging to package. Since new data centers are "green field" installation, in many cases it could be interesting to consider SDM as an alternative solution to DWDM. Solutions which use multi core to single mode patch cables have been demonstrated before [5]. However the additional cabling demands and cost eventually lead to the adoption of simpler solutions such as the PSM flavor of QSFP and QSFP28 modules [6].

In this paper we demonstrate the first time a C-band single mode, SDM VCSEL based transmitter module capable of supporting $100 \mathrm{Gbps}$ data transmission directly attached to a multicore fiber (MCF). A 1x4 VCSEL array is directly coupled to a 3D glass interposer fabricated using ultrafast laser inscription (ULI) [7], a well-established technique to embed 3D waveguides in a glass interposer. Thanks to this technique a 'fan-out' structure can be easily achieved from the linear arrangement of the VCSELs to the cylindrical geometry of the MCF [8]. Error free operation of 3 of the 4 VCSEL channels at 25 Gbps per lane is presented.

\section{FPC Assembly Processes}

The schematic of the optical transmitter is shown in Fig. 1(a). A $100 \mu \mathrm{m}$ thickness polyimide has been used for the base material, which has a dielectric constant of 3.2 and a dissipation factor of 0.002 at $1 \mathrm{MHz}$. The differential transmission lines on FPC are designed to be $100 \Omega$. A $440 \mu \mathrm{m}$ thickness FR-4 stiffener is used to enhance the stiffness of FPC and a cavity is designed for inserting the 3D glass interposer. To flip-chip bond the components, gold stud bumps are ultrasonically formed on the driver chip and on the pads of FPC for the VCSELs. The Isotropic Conductive Adhesive (ICA) epoxy H20E is dispensed on the pads of FPC and gold stud bumps for VCSELs to make connections. Then FINEPLACER flip-chip Die Bonder is used to align and connect the stud bumps and ICAepoxy $\mathrm{H} 20 \mathrm{E}$ at $150{ }^{\circ} \mathrm{C}$ for 1 hour. The fully assembled transmitter is shown in Fig. 1(b). The VCSELs used in this demonstration was designed with only $40 \mu \mathrm{m}$ square pads and in addition a plated metal perimeter making flip-chip assembly quite challenging. As a result only three of the four channels function well in this transmitter. However by removing the gold plating on the chip edges assembly and yield can be greatly improved.

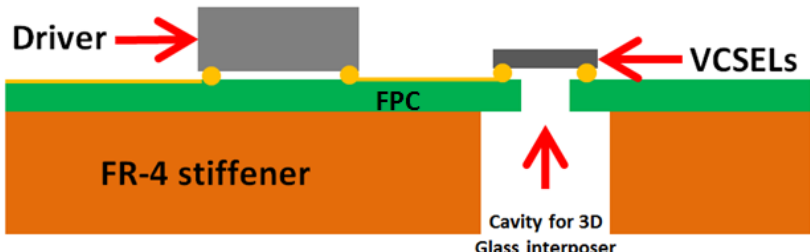

(a)

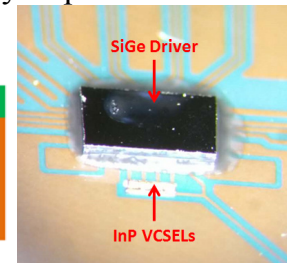

(b)

Fig. 1. (a) Schematic of the $1.5 \mu \mathrm{m}$ VCSEL based transmitter; (b) Fully assembled transmitter module.

3. Experimental Set-up and Results

As shown in Fig. 2 (a), 25 Gpbs NRZ PRBS $2^{11}-1$ electronic signal from a bit pattern generator are sent to the driver 
IC and VCSELs bonded on the FPC through an RF differential probe to characterize the performance of this transmitter. A 6-axis stage is used to control the position of the 3D glass interposer, which is shown in Fig. 2(b), in order to align the waveguides inside the glass to the apertures of VCSELs. Other two 3-axis stages are utilized to couple the light from the 4-core fiber to a SMF. This SMF is connected to an EDFA to amplify the signal and filtered to reduce the noise. After that, the optical signal is introduced to the optical spectrum analyzer, oscilloscope and error detector to verify the performance.

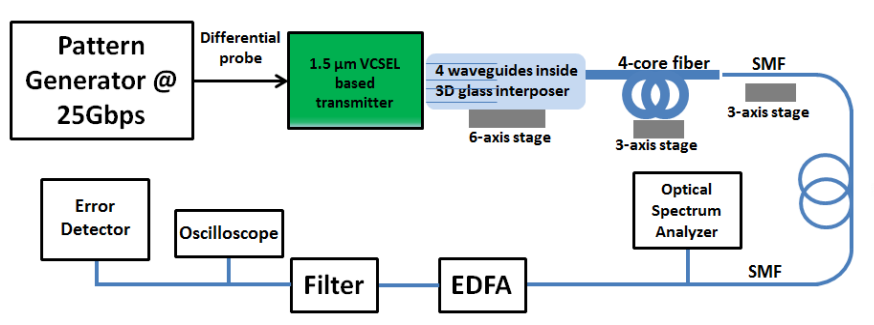

(a)

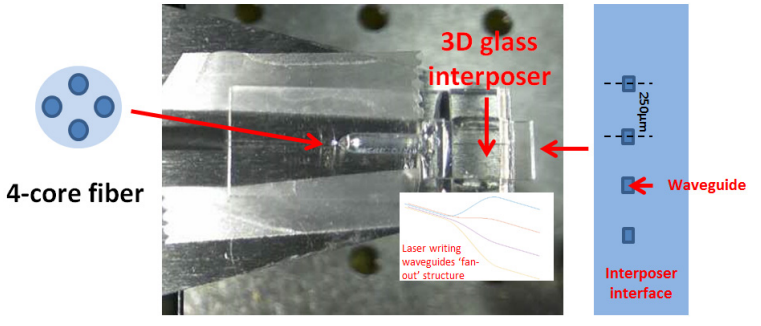

(b)

Fig. 2. (a) Experimental Set-up; (b) 3D Glass Interposer mounted on a 3-axes stage. The MCF is depicted at the left hand side and the interposer input cross-section at the right hand side. The white inset shows the fan-out design in the interposer.

$2.5 \mathrm{~V}$ low-power SiGe VCSEL driver is used and VCSELs are biased at an average current of $13 \mathrm{~mA}$. The output powers after the SMF are between $-20.3 \mathrm{dBm}$ and $-22.4 \mathrm{dBm}$ and the Signal-to-noise ratios in 3 channels are above $32 \mathrm{~dB}$. The optical spectrums are shown in Fig. 3(a). The VCSELs emit around 4.5dBm of optical power but have a numerical aperture which does not match well with that of the single mode waveguides in the glass interposer. This, in combination with the additional distance between the VSCEL aperture and the glass interposer of around $150 \mu \mathrm{m}$ due to the thickness of the FPC and the height of the stud bumps creates $18.5 \mathrm{~dB}$ of optical loss. Including lenses on the glass interposer or directly on the VCSELs can greatly improve the coupling efficiency. Visible open eyediagrams and error-free performance at 25Gbps are measured, as shown in Fig. 3(b).

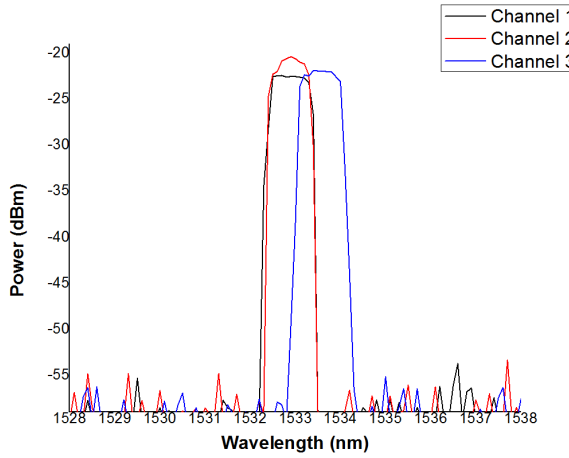

(a)

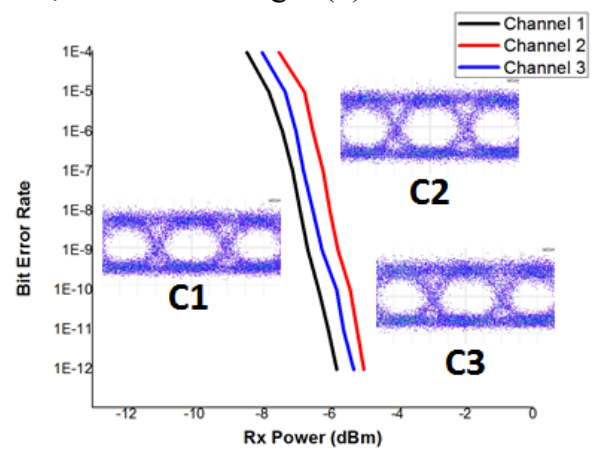

(b)

Fig. 3. (a) Optical spectra measured at the SMF; (b) Eye-diagrams and BER curves of three of the channels.

\section{Conclusion}

In this paper, we present for the first time a low cost $1.5 \mu \mathrm{m}$ InP VCSELs based $100 \mathrm{Gbps}(4 \times 25 \mathrm{Gbps})$ multicore transmitter module platform. The 3D glass interposer, which contain 4 waveguides made using ultrafast laser inscription process and a 4-core fiber is used to increase the bandwidth density. The transmitter can achieve 25Gbps error-free performance and eye diagrams are clearly open.

\section{References}

[1] Hong Liu, et. al., "Scaling Optical Interconnects in Datacenter Networks Opportunities and Challenges for WDM," 2010 18th IEEE Symposium on High Performance Interconnects

[2] Katharine Schmidtke, "Designing 100G Optical Connections" online available: https://code.facebook.com/posts/1633153936991442/designing-100g-optical-connections/

[3] Online available: $h t t p: / / m e n a r a n e t . c o m / n e w s ?$ news id $=27$

[4] Chin-Hui Chen et. al " DWDM Silicon Photonic Transceivers for Optical Interconnect "DWDM silicon photonic transceivers for optical interconnect, in Optical Interconnects Conference," in Proc. IEEE Opt. Interconnects Conf. (OI), 2015.

[5] H. Uemura et. al. " Fused taper type fan-in/fan-out device for 12 core multi-core fiber", OECC / ACOFT 2014, 6-10 July 2014, Melbourne, Australia

[6] Online available: http://www.oclaro.com/product/100g-qsfp28-lr4/

[7] Feng Chen et. al., " Optical waveguides in crystalline dielectric materials produced by femtosecond-laser micromachining",LASER\&PHOTONICS REVIEWS, 17 May 2013

[8] Dimitrios Apostolopoulos et. al., "Photonic integration enabling new multiplexing concepts in optical board-to-board and rack-to-rack interconnects” Optical Interconnects XIV 2014, San Francisco, California, United States 